\title{
Cost-effectiveness of the use of low- and high-potency statins in people at low cardiovascular risk
}

\author{
Jon Conly BA, Fiona Clement PhD, Marcello Tonelli MD SM, Brenda Hemmelgarn MD PhD, \\ Scott Klarenbach MD MSc, Anita Lloyd MSc, Finlay A. McAlister MD MSc, Don Husereau BScPharm MSc, \\ Natasha Wiebe MMath PStat, Flora Au Mec, Braden Manns MD MSc; for the Alberta Kidney Disease Network
}

See related research article by Tonelli and colleagues at www.cmaj.ca/lookup/doi/10.1503/cmaj.101280 and related commentary by Gupta at www.cmaj.ca/lookup/doi/10.1503/cmaj.111674

\begin{abstract}
Competing interests: Marcello Tonelli has received research support from Pfizer; in 2011 he served on an advisory board for Merck, providing expert opinion on global chronic kidney disease and cardiovascular disease. Brenda Hemmelgarn has received research support from Merck and Amgen. Braden Manns has received research support from Merck Canada. No competing interests declared by the other authors.
\end{abstract}

This article has been peer reviewed.

\section{Correspondence to:}

Dr. Braden Manns,

braden.manns@alberta

healthservices.ca

CMAJ 2011. DOI:10.1503 /cmaj.101281

\begin{abstract}
- ABstract
Background: Although statins have been shown to reduce the risk of cardiovascular events in patients at low cardiovascular risk, their absolute benefit is small in the short term, which may adversely affect costeffectiveness. We sought to determine the long-term cost-effectiveness (beyond the duration of clinical trials) of low- and high-potency statins in patients at low cardiovascular risk and to estimate the impact on Canada's publicly funded health care system.
\end{abstract}

Methods: Using Markov modelling, we performed a cost-utility analysis in which we compared low-potency statins (fluvastatin, lovastatin, pravastatin and simvastatin) and high-potency statins (atorvastatin and rosuvastatin) with no statins in a simulated cohort of low-risk patients over a lifetime horizon. Model outcomes included costs (in 2010 Canadian dollars), quality-adjusted life-years (QALYs) gained and the cost per QALY gained. lthough statins improve survival and reduce the risk of cardiovascular events in populations at high and moderate risk, ${ }^{1}$ their effectiveness and cost-effectiveness in lowrisk populations is less certain. ${ }^{2}$ This uncertainly is due in part to low-risk patients being less likely to have cardiovascular events over the short term. For instance, in the recent Justification for the Use of Statins in Prevention: an Intervention Trial Evaluating Rosuvastatin (JUPITER) study ${ }^{3}-\mathrm{a}$ large randomized trial comparing cardiovascular outcomes in low-risk patients randomly assigned to receive either rosuvastatin or placebo - the risk of death or nonfatal myocardial infarction over three years was $2.5 \%$ in the rosuvastatin group and 3.5\% in the placebo group, which represented a large relative, but small absolute, risk reduction in cardiovascular events.
Results: Over a lifetime horizon, the cost of managing a patient at low cardiovascular risk was estimated to be about $\$ 10100$ without statins, \$15 200 with low-potency statins and $\$ 16400$ with high-potency statins. The cost per QALY gained with high-potency statins (v. no statins) was $\$ 21300$; the use of lowpotency statins was not considered economically attractive. These results were robust to sensitivity analyses, although their use became economically unattractive when the duration of benefit from statin use was assumed to be less than 10 years.

Interpretation: Use of high-potency statins in patients at low cardiovascular risk was associated with a cost per QALY gained that was economically attractive by current standards, assuming that the benefit from statin use would continue for at least 10 years. However, the overall expenditure on statins would be substantial, and the ramifications of this practice should be carefully considered by policy-makers.
Other cholesterol-lowering interventions are available, such as diet, exercise and the use of other hypolipidemic agents, but the use of statins is the only such intervention known to reduce cardiovascular risk in people with low and high blood cholesterol levels. ${ }^{4-7}$ Thus, statins are now primarily indicated for the reduction of cardiovascular risk instead of being used mainly for the management of hypercholesterolemia. ${ }^{8}$

With this broadening indication for use, expenditures on statins have increased and represent about $13 \%$ of total expenditures by provincial formularies in Canada. ${ }^{9}$ The absolute number of people at low cardiovascular risk who are taking statins has increased substantially over the last decade, driven by the large number of low-risk people in the general population..$^{10}$ In addition, statins that are more effective in lowering low- 
density lipoprotein (LDL) cholesterol levels have become available., ${ }^{3,11}$ These high-potency statins (atorvastatin and rosuvastatin) are substantially more expensive than low-potency statins available as generics (pravastatin, simvastatin, fluvastatin and lovastatin), although atorvastatin has recently become available as a generic in Canada. ${ }^{12}$ Increasing costs and concerns over the absolute benefit of statins in people at low cardiovascular risk has raised concerns about the costeffectiveness of statins in this group.

We performed an incremental cost-utility analysis comparing low- and high-potency statins with no statins in patients at low cardiovascular risk in a Canadian setting. We used findings from our group's recent systematic review of the efficacy of statins for primary prevention in low-risk people ${ }^{13}$ as well as observational data from a large provincial registry of patients documenting existing statin use. Our objective was to determine which strategy represents the best use of health care resources for the publicly funded health care system, and what investment would be required to fund statins.

\section{Methods}

\section{Cost-utility analysis}

The methods are described in detail in Appendix 1 (available at www.cmaj.ca/lookup/suppl
/doi:10.1503/cmaj.101281/-/DC1). In brief, we performed an incremental cost-utility analysis comparing low- and high-potency statins with no statins in patients at low cardiovascular risk. We defined low cardiovascular risk as a 10 -year risk of less than $20 \%$ for cardiovascular-related death or nonfatal myocardial infarction, which approximates the risk among patients without cardiovascular disease and diabetes at baseline. Treatment strategies included no statins, the use of low-potency statins (fluvastatin, lovastatin, pravastatin and simvastatin) or the use of highpotency statins (atorvastatin and rosuvastatin).

For the primary analysis, we used Markov modelling of a patient cohort at low cardiovascular risk over a lifetime horizon (Figure 1), discounting costs and health outcomes at $5 \% .{ }^{14}$ The model included clinical states reflective of the outcomes reported in the randomized trials included in the systematic review used for our analysis, ${ }^{13}$ such as death from any cause, nonfatal stroke, nonfatal myocardial infarction and unstable angina necessitating hospital admission. Because adverse events or intolerance to statins can develop, or because patients may simply stop using statins, we included health states where patients stopped using statins (Figure 1). Model outcomes included costs (in 2010 Canadian dollars), quality-adjusted life-years (QALYs) gained and the cost per QALY gained.

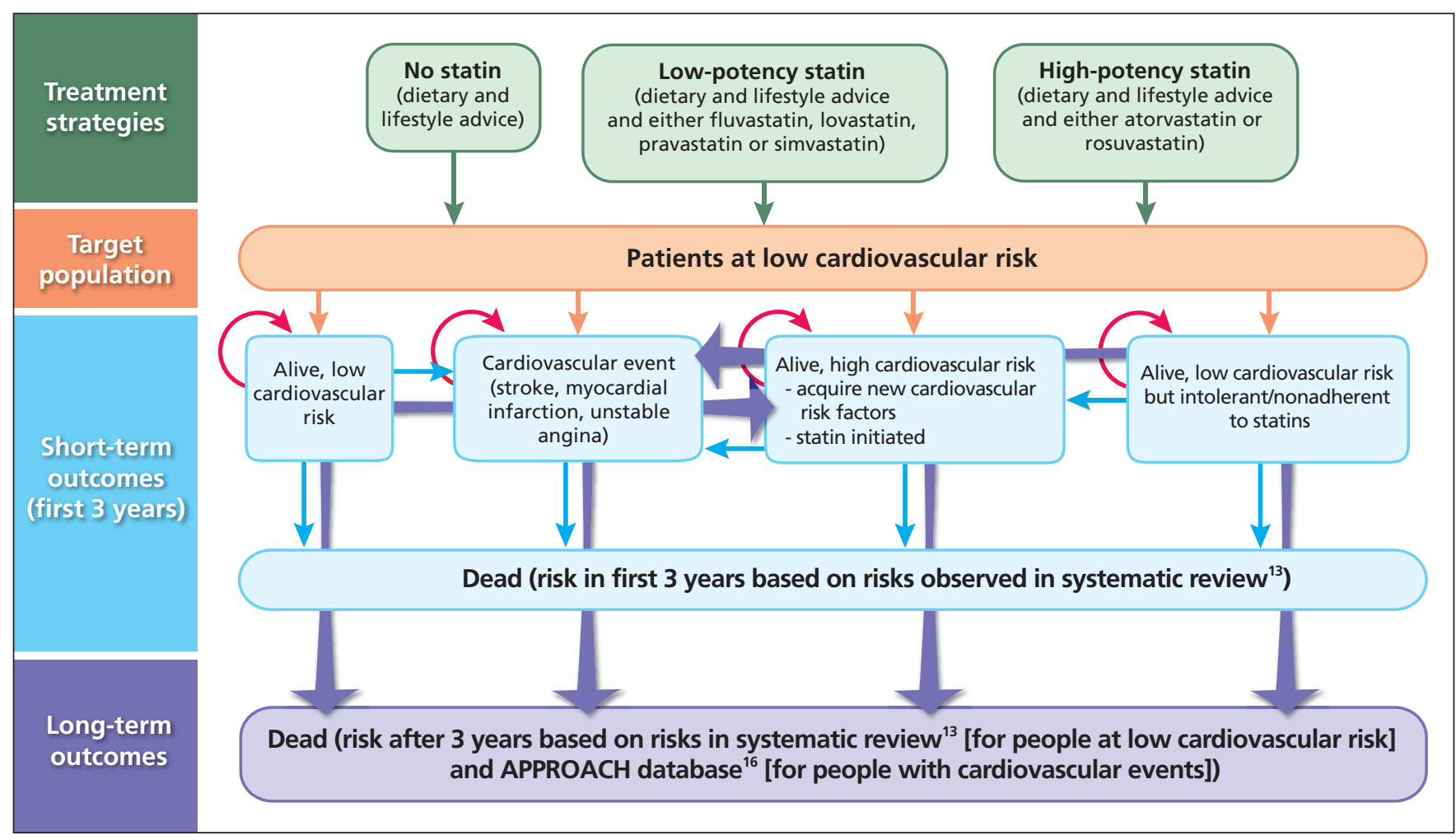

Figure 1: Structure of the Markov model, showing the flow of patients and outcomes that were considered in the short and long term in this cost-utility analysis. 
The model covered two periods. For the first three years (the mean duration of the clinical trials in our systematic review), the model replicated the findings from our systematic review, ${ }^{13}$ basing the risk of cardiovascular events and death on the findings from the review for each treatment strategy (Table 1). After the first three-year period, patients who did not have a cardiovascular event were assumed to continue free from cardiovascular disease, with ongoing risks of cardiovascular events and death based on the findings from our systematic review. For patients in whom nonfatal cardiovascular events developed, we based their subsequent risk of death, cardiovascular events and use of health care resources on a large observational provincial registry (Alberta Provincial Project for Outcome Assessment in Coronary Heart [APPROACH] disease database). This database tracks health care outcomes, such as cardiovascular-related events and death, in patients with a first cardiovascular event, with outcomes available for up to 15 years. Health care resources and estimates of patients' utility (a measure of overall quality of life) were based on data from the published literature and the APPROACH database (Table 2, and eTable 1 in Appendix 1, available at www.cmaj.ca/lookup /suppl/doi:10.1503/cmaj.101281/-/DC1).

Consistent with published guidelines, ${ }^{14}$ we established face and internal validity of our model by comparing model outputs (a function of both input variables and model structure) for the no-statin and statin arms with reported data from the meta-analysis. ${ }^{13}$ We investigated predictive validity of the model by comparing life expectancy estimated by the model against the mean life expectancy of similarly aged people reported by Statistics Canada. ${ }^{20}$ We performed all analyses using Treeage Pro 2009 (Treeage Software, Inc.).

\section{Budget impact analysis}

We used data from the 2007 Canadian Community Health Survey ${ }^{21}$ and the National Health and Nutrition Examination Survey ${ }^{22}$ to estimate the proportion of Canadians who might fit the following criteria for low cardiovascular risk. We used various definitions ranging from very low to low risk: (a) men and women over the age of 40 without heart disease, diabetes or stroke; (b) men over 50 and women over 60 without heart disease, diabetes or stroke; and (c) men over 50 and women over 60 without heart disease, diabetes or stroke but who either had hypertension or were smokers. We used these proportions applied to the Canadian population to estimate annual drug costs assuming different usage of low- and high-potency statins.

\section{Results}

\section{Model validity}

When we compared the proportion of patients who died or experienced nonfatal myocardial infarction or nonfatal stroke in the three longest randomized trials in our systematic review ${ }^{23-25}$ with our model outputs at five years using input data from each of these trials separately (i.e., a test of internal validity), we noted agreement for all outcomes $\left(R^{2}>0.99\right)$. Moreover, our model predicted a mean undiscounted life expectancy of 20.5 years for patients receiving no statins, 21.0 years for patients receiving low-potency statins and 21.4 years for patients receiving high-

Table 1: Rate of clinical events among patients not receiving statins, and relative risk of events associated with the use of low- and high-potency statins in patients at low cardiovascular risk*

\begin{tabular}{|c|c|c|c|}
\hline Outcome & $\begin{array}{c}\text { Annual rate of events } \\
\text { in no-statin arm }(95 \% \mathrm{Cl})\end{array}$ & $\begin{array}{l}\text { RR for low-potency statin } \\
\text { v. no statin }(95 \% \mathrm{Cl})\end{array}$ & $\begin{array}{l}\text { RR for high-potency statin } \\
\text { v. no statin }(95 \% \mathrm{Cl})\end{array}$ \\
\hline Death from any cause & $0.0075 \quad(0.0062-0.0093)$ & $0.90(0.79-1.03)$ & $0.85(0.74-0.96)$ \\
\hline Nonfatal stroke & $0.0041 \quad(0.0032-0.0053)$ & $0.88(0.73-1.06)$ & $0.51(0.33-0.79)$ \\
\hline Unstable angina & $0.0020 \quad(0.0014-0.0028)$ & $0.70(0.51-0.97)$ & $0.73(0.48-1.11)$ \\
\hline Rhabdomyolysis & $0.00012(0.00001-0.00042)$ & $0.50(0.05-5.51)$ & $2.99(0.31-28.75)$ \\
\hline \multicolumn{4}{|c|}{$\begin{array}{l}\text { Note: } \mathrm{Cl}=\text { confidence interval, } \mathrm{RR}=\text { relative risk. } \\
\text { *Data are taken from a recent systematic review; "low cardiovascular risk" was defined in the systematic review as a } 10 \text {-year risk of cardiovascular-related death } \\
\text { or nonfatal myocardial infarction of less than } 20 \% .^{18} \\
\text { tDefined in the reference case as the development of diabetes. } \\
\text { fBecause few trials of low-potency statins reported the development of diabetes, we assumed the annual rate to be similar to that reported in trials of high- } \\
\text { potency statins. }\end{array}$} \\
\hline
\end{tabular}


potency statins. As expected, these values were slightly lower than the mean life expectancy for Canadians of similar age reported by Statistics Canada (men 21.7 years, women 25.6 years), ${ }^{20}$ most of whom would be at even lower cardiovascular risk than people included in our model.

\section{Cost estimates}

Over a lifetime horizon, the cost of managing a patient at low cardiovascular risk was estimated to be about \$10 100 without statins, \$15 200 with low-potency statins and \$16 400 with highpotency statins (Table 3 ). Compared with no statins, the incremental gain was 0.17 QALYs with low-potency statins and 0.30 QALYs with high-potency statins. The cost per QALY gained was \$21300 with high-potency statins versus no statins. The cost per QALY gained with lowpotency statins versus no statins was less attractive than this, which suggested that the use of lowpotency statins would not be an economically attractive strategy in the reference case (Table 3).

\section{Sensitivity analyses}

Plausible variations in utility estimates, costs or the effect of statin treatment on adverse events

Table 2: Cost of statins and of managing clinical events in the reference case*

\begin{tabular}{|c|c|c|c|}
\hline Variable & $\begin{array}{l}\text { Base case } \\
\text { estimate, } \$ \dagger\end{array}$ & $\begin{array}{l}\text { Range } \\
(95 \% \mathrm{Cl}) \ddagger\end{array}$ & Source \\
\hline Annual cost of low-potency statin§ & 527 & NA & $\begin{array}{l}\text { Provincial drug } \\
\text { benefit list }^{12}\end{array}$ \\
\hline Annual cost of high-potency statin§ & 790 & NA & $\begin{array}{l}\text { Provincial drug } \\
\text { benefit list }^{12}\end{array}$ \\
\hline $\begin{array}{l}\text { Annual cost of laboratory monitoring for a } \\
\text { patient taking a statinף }\end{array}$ & 109 & NA & $\begin{array}{l}\text { AHS laboratory } \\
\text { costs }\end{array}$ \\
\hline $\begin{array}{l}\text { Cost of managing a patient with mild/moderate } \\
\text { stroke (Rankin score } 0-3 \text { ) in year } 1^{* *}\end{array}$ & 3222 & $698-7142$ & Berthiaume $^{15}$ \\
\hline $\begin{array}{l}\text { Cost of managing a patient with severe stroke } \\
\text { (Rankin score } 4-6 \text { ) in year } 1^{* *}\end{array}$ & 48858 & $9942-107658$ & Berthiaume ${ }^{15}$ \\
\hline $\begin{array}{l}\text { Annual cost of managing a stroke survivor in } \\
\text { years } 2 \text { and } 3+\dagger\end{array}$ & 6930 & $\pm 25 \%$ & Berthiaume $^{15}$ \\
\hline $\begin{array}{l}\text { Cost of managing a patient with nonfatal } \\
\text { myocardial infarction in year } 1\end{array}$ & 4591 & $4362-4648$ & APPROACH ${ }^{16}$ \\
\hline $\begin{array}{l}\text { Annual cost of managing a patient with nonfatal } \\
\text { myocardial infarction in years } 2 \text { and } 3\end{array}$ & 1353 & $1170-1536$ & APPROACH ${ }^{16}$ \\
\hline $\begin{array}{l}\text { Cost of managing a patient with unstable angina } \\
\text { in year } 1\end{array}$ & 4164 & $3972-4356$ & APPROACH ${ }^{16}$ \\
\hline $\begin{array}{l}\text { Annual cost of managing a patient with unstable } \\
\text { angina in years } 2 \text { and } 3\end{array}$ & 3419 & $3057-3779$ & APPROACH ${ }^{16}$ \\
\hline Cost of coronary artery bypass grafting $\neq \ddagger$ & 17925 & $\pm 25 \%$ & APPROACH ${ }^{16}$ \\
\hline Cost of percutaneous coronary intervention $\ddagger \ddagger$ & 7380 & $\pm 25 \%$ & APPROACH ${ }^{16}$ \\
\hline $\begin{array}{l}\text { Annual cost of managing a long-term survivor } \\
\text { of a cardiovascular event (in year } 4 \text { and } \\
\text { beyond)§§ }\end{array}$ & 3682 & $130-5352$ & APPROACH ${ }^{16}$ \\
\hline $\begin{array}{l}\text { Annual cost of managing a patient whose } \\
\text { cardiovascular risk status increasesףी }\end{array}$ & 2406 & $50-3770$ & APPROACH ${ }^{16}$ \\
\hline Cost of managing a patient with rhabdomyolysis & 78840 & $\pm 25 \%$ & Skrabal et al. ${ }^{17}$ \\
\hline \multicolumn{4}{|c|}{ 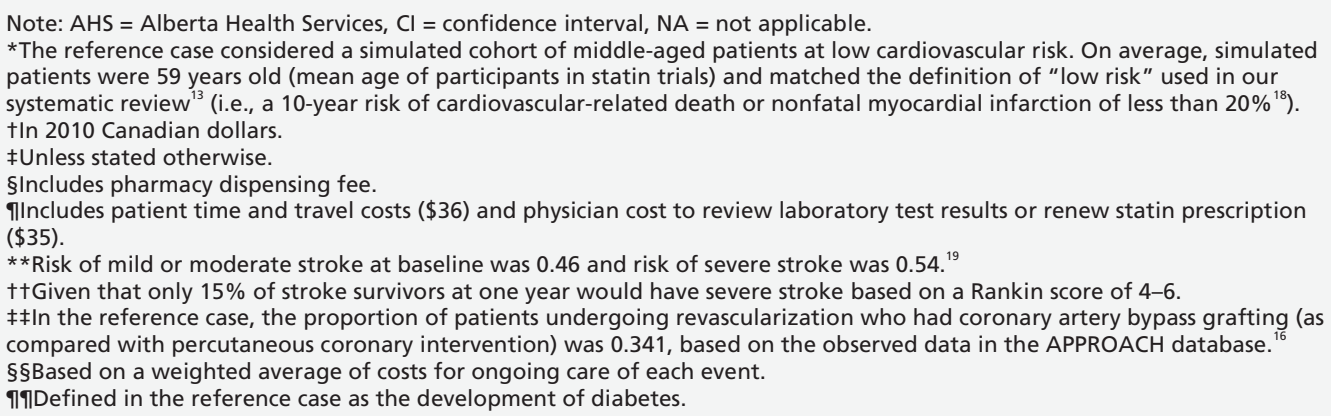 } \\
\hline
\end{tabular}


(including the risk of new diabetes) did not appreciably affect the estimated cost per QALY gained (Table 4). Changes in the relative risk estimates of all clinical events (upper and lower 95\% confidence intervals for death, nonfatal myocardial infarction, nonfatal stroke and unstable angina) led to substantial variations in the estimated cost per QALY gained: ranging from $\$ 10500$ per QALY to $\$ 109000$ per QALY with the use of high-potency statins versus no statins. In a sensitivity analysis that considered a generic price for atorvastatin and that assumed similar efficacy between atorvastatin and high-potency statins (i.e., using the relative risk for cardiovascular events and death for high-potency statins as a class), the use of generic atorvastatin was cost saving and improved outcomes compared with the use of low-potency statins.

When we considered an alternate definition of low risk - a 10-year risk of cardiovascularrelated death or nonfatal myocardial infarction less than $10 \%{ }^{8}$ - the cost per QALY gained (\$22 100) was similar to the finding from the primary analysis.

Given that the results of the JUPITER study ${ }^{3}$ had a strong influence on the results of the metaanalysis, ${ }^{13}$ we completed an analysis in which we excluded the relative risk estimates from the JUPITER trial. We found that low-potency statins were no longer economically unattractive (i.e., they were no longer removed by extended dominance), and their use was associated with a cost per QALY gained of \$27 600 compared with no statins; use of high-potency statins (v. low-potency statins) was associated with a cost per QALY gained of $\$ 31300$.

We modelled three different scenarios that might be expected to adversely affect the costeffectiveness of statin use in low-risk people: variation in the duration of benefit from statin use, variation in the increased risk of transition to a higher cardiovascular risk status (by acquiring new vascular risk factors), and statin use in people at even lower cardiovascular risk.

As the duration of benefit associated with statin use decreased, the cost per QALY gained became less attractive (Table 4). When the duration of benefit from statins was assumed to be only three years, the cost per QALY gained with high-potency statins versus no statins was $\$ 353500$ (Table 4). Both high- and low-potency statins became more economically attractive as the assumed duration of benefit increased above 10 years.

Increasing the annual risk of transitioning to a higher cardiovascular risk status (prompting statin use) led to a less attractive cost per QALY gained. However, even when the annual risk was assumed to be $10 \%$ (likely unrealistically high), the cost per QALY gained with high-potency statins compared with no statin use was \$53 200 .

As expected, increasing the annual cardiovascular risk (the combined risk of nonfatal myocardial infarction or cardiovascular-related death) resulted in treatment with high-potency statins (v. no statins) becoming more economically attractive. Alternatively, decreasing the annual cardiovascular risk to $0.25 \%$ resulted in the cost per QALY becoming less attractive, at $\$ 40100$ per QALY for treatment with high-potency statins (Table 4).

\section{Probabilistic sensitivity analyses}

Probabilistic sensitivity analysis showed that the use of high-potency statins, compared with no statins, resulted in higher costs and more QALYs gained in the majority of simulations (Appendix 1, eFigure 1, available at www.cmaj.ca/lookup /suppl/doi:10.1503/cmaj.101281/-/DC1). In addition, the majority of points $(94.8 \%)$ compar-

Table 3: Cost of treatment strategies over a lifetime in the reference case*

\begin{tabular}{|c|c|c|c|c|c|}
\hline Treatment strategy & Cost, $† \$$ & $\begin{array}{c}\text { Incremental } \\
\text { cost, } \$\end{array}$ & $\begin{array}{c}\text { Effectiveness, } † \\
\text { QALYs }\end{array}$ & $\begin{array}{l}\text { Incremental } \\
\text { effectiveness, } \\
\text { QALYs gained }\end{array}$ & $\begin{array}{c}\text { Incremental cost per } \\
\text { QALY gained, † \$ }\end{array}$ \\
\hline No statin & 10100 & - & 11.18 & - & - \\
\hline Low-potency statin & 15200 & 5100 & 11.35 & 0.17 & $\begin{array}{c}\text { Eliminated by } \\
\text { extended dominance }\end{array}$ \\
\hline High-potency statin & 16400 & $6300 \S$ & 11.48 & $0.30 \S$ & $21300 \S$ \\
\hline \multicolumn{6}{|c|}{$\begin{array}{l}\text { Note: QALY = quality-adjusted life-year; costs are in } 2010 \text { Canadian dollars. } \\
\text { *The reference case considered a simulated cohort of middle-aged patients at low cardiovascular risk. On average, simulated } \\
\text { patients were } 59 \text { years old (the mean age of participants in the statin trials) and matched the definition of "low risk" used in } \\
\text { our systematic review }{ }^{13} \text { (i.e., a } 10 \text {-year risk of cardiovascular-related death or nonfatal myocardial infarction of }<20 \%{ }^{18} \text { ). } \\
\text { †Over a lifetime horizon. } \\
\text { †Although the use of low-potency statins is associated with better outcomes (at higher cost) compared with no statin use, this } \\
\text { strategy was eliminated from consideration by extended dominance, because a QALY can be purchased at a lower cost with a } \\
\text { high-potency statin. } \\
\S \text { Compared with no statin. }\end{array}$} \\
\hline
\end{tabular}


Table 4: Sensitivity analyses of the impact of plausible variations in utility estimates, costs or effect of statin treatment on adverse events on incremental cost-effectiveness ratios for low- and high-potency statins in people at low cardiovascular risk*

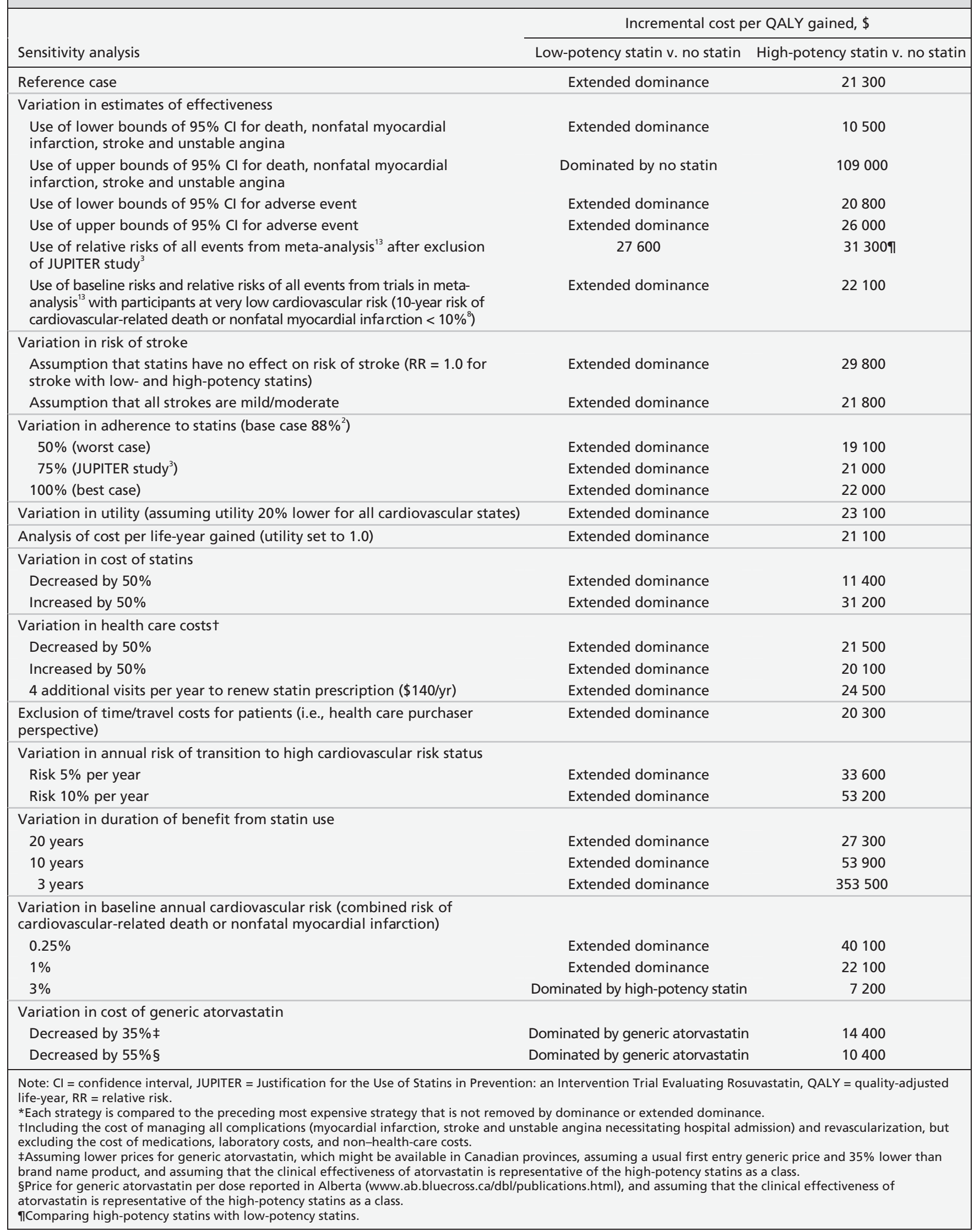


ing high-potency statins with no statins fell below an arbitrary willingness-to-pay threshold of \$50 000 per QALY (Appendix 1, eFigure 1). At a willingness-to-pay threshold of $\$ 25000$ per QALY gained, the likelihood that high-potency statin treatment would be cost-effective, compared with the next most expensive strategy, was $54.8 \%$ (Appendix 1, eFigure 2). The likelihood increased to $72.2 \%$ at a willingness-to-pay threshold of $\$ 50000$ per QALY gained, and to $75.9 \%$ at a willingness-to-pay threshold of $\$ 100000$ per QALY gained.

\section{Budget impact}

The annual drug costs for using statins in various low-risk populations in Canada without diabetes or cardiovascular disease are shown in Appendix 1 (eTable 2). Using the most restrictive definition of low risk (men over the age of 50 and women over 60 without diabetes, heart disease or stroke but who either have hypertension or are current smokers), we would expect an additional 4.03 million people to be eligible for statin therapy if the treatment strategy involved statin use regardless of the person's LDL cholesterol level. If treatment were limited to people with an LDL cholesterol level greater than $4.5 \mathrm{mmol} / \mathrm{L}$, the eligible population would decrease to 374014 (Appendix 1, eTable 2).

Using the most liberal definition of low risk (men and women over the age of 40 without diabetes, heart disease or stroke, regardless of the presence of hypertension or smoking status), we would expect an additional 1.1-11.6 million people to be eligible for statin therapy, depending on whether treatment was given regardless of the LDL cholesterol level or whether LDL cholesterol levels were used to target therapy (Appendix 1, eTable 2). In this unlikely scenario, expenditures on statins would increase by $\$ 9.17$ billion annually in Canada.

\section{Interpretation}

We found that use of high-potency statins compared with no statins was associated with a cost per QALY gained of $\$ 21300$. In the reference case and in most of the sensitivity analyses, lowpotency statins were not considered economically attractive, since a QALY could be purchased at a lower cost with high-potency statins than with low-potency statins. In general, our base-case results were robust to plausible variation in stochastic variables, except in analyses that limited the duration of benefit from statins to less than 10 years. The cost per QALY gained of \$21 300 may seem more attractive than would be expected given the relatively high number needed to treat (239 for preventing one death based on the absolute risk reduction in mortality taken from our systematic review $\left.{ }^{13}\right)$. However, the number needed to treat was calculated from trials in which the median duration of follow-up was two years, whereas our analysis assumed that statins would continue to reduce the risk of cardiovascular events over the patient's lifetime.

Given the projected rate of growth in expenditures, the annual drug expenditure in Canada in 2009 is expected to be nearly $\$ 30.0$ billion. $^{9}$ Expanding the use of statins to a low-risk population could result in a budgetary increase of $\$ 295$ million to $\$ 9.2$ billion annually depending on the population and the LDL cholesterol used to target therapy. Although the cost per QALY gained is within a range that may be perceived as a reasonable use of resources, the money directed toward statin treatment will need to come from elsewhere in the government budget: either a redirection of health care resources currently being used to fund other programs and interventions, or a redirection of funds being used elsewhere in the social system.

Previous studies have shown that statins are cost-effective (and cost saving in many subgroups) in the secondary prevention of cardiovascular disease. ${ }^{2.26}$ Even among higher risk patients without documented vascular disease, the use of statins has been associated with a cost per QALY gained below $\$ 20000$, which is considered by many to represent good value for money in publicly funded health care systems. ${ }^{27-29}$ However, whether statins are cost-effective in people at very low cardiovascular risk has been uncertain. ${ }^{2,30}$ Some studies have suggested that their use in such people was associated with a cost per QALY gained of less than $£ 20000,{ }^{2}$ but only under the assumption that statin use reduces the risk of stroke, which our clinical systematic review suggests. ${ }^{13}$ In a study involving low-risk Americans, in which the benefit from statins in low-risk people was extrapolated from the impact of statins on cholesterol levels, Pletcher and colleagues noted an incremental cost of US\$42000 per QALY gained. ${ }^{30}$

\section{Limitations}

Our analysis has limitations. We modelled lowand high-potency statins separately, although neither metaregression nor indirect comparisons showed conclusive evidence that use of highpotency statins was clinically superior to treatment with low-potency statins. ${ }^{13}$ However, indirect comparisons showed consistent but nonsignificant trends to reductions in mortality and cardiovascular outcomes associated with treatment with highpotency statins.

Our estimate of long-term survival for patients with cardiovascular events was taken from an 
observational cohort of patients who underwent catheterization, thereby excluding patients who do not undergo catheterization. However, the effect of this bias was likely minimal because our model was robust to changes in these survival estimates. Treating patients without a "disease" with a medication to reduce risk raises issues about the impact of medication use on quality of life. If future evidence suggests that statins negatively affect quality of life, this should be incorporated in future economic analyses.

Another potential limitation is that our effectiveness results included data from the recent JUPITER trial, ${ }^{3}$ which contributed significantly to the overall reduction in risk observed with a highpotency statin strategy. Although the validity of this trial has been questioned, ${ }^{4}$ even when we excluded the JUPITER trial from our analysis, the results were largely similar, as were the results from analyses in which we used various definitions of low cardiovascular risk. Our analysis hinges upon the assumption that statins continue to reduce the risk of cardiovascular events over the patient's lifetime. In support of this assumption, we found no suggestion that the efficacy of statins was lower in the longer term compared with the efficacy in short-term randomized studies.

\section{Conclusion}

Use of high-potency statins in patients at low cardiovascular risk was associated with a cost per QALY gained that appeared to be economically attractive by current standards, assuming that statin use would continue to effectively reduce the risk of cardiovascular events and death for at least 10 years after initial prescription. High-potency statins may be more attractive than low-potency ones, although definitive evidence of their superior efficacy is lacking. On the basis of our findings, it appears that provinces should continue to fund the use of all statins in a similar fashion, regardless of potency, for use in patients at low cardiovascular risk. However, the overall expenditure on statins in low-risk populations would be substantial, and the ramifications of this practice should be carefully considered by policy-makers.

\section{References}

1. van Boven AJ, Brugemann J, de Graeff PA, et al. The 4S study Implications for prescribing. Drugs 1996;51:507-14.

2. Ward S, Lloyd Jones M, Pandor A, et al. A systematic review and economic evaluation of statins for the prevention of coronary events. Health Technol Assess 2007;11:1-160.

3. Ridker PM, Danielson E, Fonseca FA, et al.; JUPITER Study Group. Rosuvastatin to prevent vascular events in men and women with elevated C-reactive protein. N Engl J Med 2008;359: 2195-207.

4. de Lorgeril M, Salen P, Abramson J, et al. Cholesterol lowering, cardiovascular diseases, and the rosuvastatin-JUPITER controversy: a critical reappraisal. Arch Intern Med 2010;170:1032-6.

5. Green LA. Cholesterol-lowering therapy for primary prevention: still much we don't know. Arch Intern Med 2010;170:1007-8.
6. Kaul S, Morrissey RP, Diamond GA. By Jove! What is a clinician to make of JUPITER? Arch Intern Med 2010;170:1073-7.

7. Ray KK, Seshasai SR, Erqou S, et al. Statins and all-cause mortality in high-risk primary prevention: a meta-analysis of 11 randomized controlled trials involving 65229 participants. Arch Intern Med 2010;170:1024-31.

8. Genest J, McPherson R, Frohlich J, et al. 2009 Canadian Cardiovascular Society/Canadian guidelines for the diagnosis and treatment of dyslipidemia and prevention of cardiovascular disease in the adult - 2009 reccommendations. Can J Cardiol 2009;25: 567-79.

9. Canadian Institute for Health Information. Drug expenditure in Canada, 1985 to 2009. Ottawa (ON): The Institute; 2010.

10. Raymond CB, Morgan SG, Katz A, et al. A population-based analysis of statin utilization in British Columbia. Clin Ther 2007;29:2107-19.

11. Nissen SE, Nicholls SJ, Sipahi L, et al. Effect of very highintenisty statin therapy on regression of coronary atherosclerosis: The ASTEROID Trial. JAMA 2006;295:1556-65.

12. Alberta Health and Wellness drug benefit list: pricing policy. Edmonton (AB): Alberta Health and Wellness; 2010.

13. Tonelli M, Lloyd A, Clement F, et al. Efficacy of statins for primary prevention in people at low cardiovascular risk: a metaanalysis. CMAJ 2011;183:E1189-1202.

14. Canadian Agency for Drugs and Technologies in Health. Guidelines for the economic evaluation of health technologies: Canada 3rd ed. Ottawa (ON): The Agency; 2006.

15. Berthiaume L. A cost-utility analysis of decompressive hemicraniectomy in pateints with malignant middle cerebral artery infarction. University of Calgary [thesis]; 2009.

16. Davies RA. Overview of 'APPROACH' - the Alberta Provincial Program for Outcome Assessment in Coronary Heart Disease. Can J Cardiol 2000;16:1222-4.

17. Skrabal MZ, Stading JA, Cannella CA, et al. Two cases of rhabdomyolysis associated with high-dose simvastatin. Am J Health Syst Pharm 2003;60:578-81.

18. Grundy SM, Cleeman JI, Merz CN, et al. Implications of recent clinical trials for the National Cholesterol Education Program Adult Treatment Panel III Guidelines. J Am Coll Cardiol 2004; 44:720-32.

19. Kalra L, Evans A, Perez I, et al. Alternative strategies for stroke care: a prospective randomized controlled trial. Lancet 2000; 356:894-9.

20. Deaths and mortality rates, by age group and sex, Canada, provinces and territories, annual. CANSIM Table 102-0504. Ottawa (ON): Statistics Canada; 2011.

21. Canadian community health survey (CCHS): annual component user guide. Ottawa (ON): Statistics Canada; 2009.

22. Muntner P, Mann D, Razzouk L, et al. Is measuring C-reactive protein useful for guiding treatment in women $>$ or $=60$ years and men $>$ or $=50$ years of age? Am J Cardiol 2009;104:354-8.

23. Downs JR, Clearfield M, Tyroler HA, et al. Air Force/Texas Coronary Atherosclerosis Prevention Study (AFCAPS/TEXCAPS): additional perspectives on tolerability of long-term treatment with lovastatin. Am J Cardiol 2001;87:1074-9.

24. ALLHAT Officers and Coordinators for the ALLHAT Collaborative Research Group. The Antihypertensive and Lipid-Lowering Treatment to Prevent Heart Attack Trial. Major outcomes in moderately hypercholesterolemic, hypertensive patients randomized to pravastatin vs usual care: The Antihypertensive and Lipid-Lowering Treatment to Prevent Heart Attack Trial (ALLHAT-LLT). JAMA 2002;288:2998-3007.

25. Nakamura $\mathrm{H}$, Arakawa $\mathrm{K}$, Itakura $\mathrm{H}$, et al. Primary prevention of cardiovascular disease with pravastatin in Japan (MEGA Study): a prospective randomised controlled trial. Lancet 2006;368:1155-63.

26. Johannesson M, Jonsson M, Kjekshus J, et al. Cost effectiveness of simvastatin treatment to lower cholesterol levels in patients with coronary heart disease. N Engl J Med 1997;336:332-6.

27. Laupacis A, Feeny D, Detsky AS, et al. How attractive does a new technology have to be to warrant adoption and utilization? Tentative guidelines for using clinical and economic evaluations. CMAJ 1992;146:473-81.

28. Mihaylova B, Briggs A, Armitage J, et al.; Heart Protection Study Collaborative. Lifetime cost effectiveness of simvastatin in a range of risk groups and age groups derived from a randomised trial of 20,536 people. BMJ 2006;333:1145.

29. National Institute for Health and Clinical Excellence. Social value judgements: principles for the development of NICE guidance. 2nd ed. The Institute; 2008. Available: www.nice.org.uk /media/C18/30/SVJ2PUBLICATION2008.pdf (accessed 2008 Nov. 10).

30. Pletcher MJ, Lazar L, Bibbins-Domingo K, et al. Comparing impact and cost-effectiveness of primary prevention strategies for lipid-lowering. Ann Intern Med 2009;150:243-54. 
Affiliations: From the Alberta Kidney Disease Network (Conly, Tonelli, Hemmelgarn, Klarenbach, Lloyd, Wiebe, $\mathrm{Au}$ ), Calgary and Edmonton, Alta.; the Departments of Medicine (Clement, Hemmelgarn, Manns) and Community Health Sciences (Hemmelgarn, Manns), University of Calgary, Calgary, Alta.; the Department of Medicine (Tonelli, Klarenbach, Lloyd, McAlister, Wiebe), University of Alberta, Edmonton, Alta.; the Libin Cardiovascular Institute (Manns), University of Calgary, Calgary, Alta.; and the Department of Epidemiology and Community Medicine (Husereau), University of Ottawa, Ottawa, Ont. The complete list of members of the Alberta Kidney Disease Network is available at www.akdn.info.

Contributors: All of the authors contributed to the conception and design of the study. Marcello Tonelli, Fiona Clement, Braden Manns, Anita Lloyd and Jonathan Conly contributed to the acquisition of data and drafted the report. All of the authors contributed to the analysis and interpretation of data, critically revised the report for important intellectual content and approved the final version submitted for publication.

Funding: This study was jointly funded by the Canadian Agency for Drugs and Technology in Health and the Alberta
Heritage Foundation for Medical Research Interdisciplinary Team Grants Program (which supports the Interdisciplinary Chronic Disease Collaboration). The study sponsors had no role in the design of the study, the collection, analysis or interpretation of data, the writing of the report or the decision to submit the article for publication.

Acknowledgements: The authors thank Dr. Steven Grover for his helpful comments on an earlier draft of this manuscript.

Marcello Tonelli, Finlay McAlister and Braden Manns are supported by Alberta Innovates - Health Solutions (formerly the Alberta Heritage Foundation for Medical Research (AHFMR) Health Scholar Awards. Brenda Hemmelgarn and Scott Klarenbach were supported by Population Health Investigator Awards from Alberta Innovates - Health Solutions. Marcello Tonelli was supported by a Government of Canada Research Chair in the optimal care of people with chronic kidney disease. Fiona Clement was supported by a postdoctoral fellowship award from the Canadian Health Services Research Foundation and AHFMR. Marcello Tonelli, Brenda Hemmelgarn, Scott Klarenbach, Finlay McAlister and Braden Manns were supported by an alternative funding plan from the Government of Alberta and the Universities of Alberta and Calgary. 